\title{
Personality changes in Huntington's Disease
}

\author{
M. Mendonça Bettencourt', F. Ramalho e Silva². \\ 1Unidade Local de Saúde do Nordeste, Departamento de Psiquiatria e Saúde Mental, Bragança, Portugal. \\ 2Unidade Local de Saúde de Matosinhos, Departamento de Saúde Mental, Porto, Portugal.
}

\section{Introduction}

Huntigton's Disease (HD) is an autosomal dominant disorder that results from an expansion in the CAG repeat within exon 1 of the gene encoding huntingtin. Mutant protein toxicity is the most probable pathological process, involving mainly the central nervous system, with atrophy of the caudate and putamen. Its predominant clinical features are choreiform movements, psychiatric symptoms and dementia.

\section{Psychiatric Manifestations of Huntington's Disease}

Several neuropsychiatric symptoms may be associated or precede motor symptoms in HD. Cognitive and behavioural symptoms can appear at least 15 years prior to formal motor diagnosis. This knowledge has given rise to a description of a HD prodrome in at-risk individuals that also includes subtle motor symptoms, electrophysiologic alterations and basal ganglia atrophy.

Patients with HD may present mood changes, with irritability, depression, anxiety, apathy, impaired emotional recognition, lack of empathy, obsessive thinking, changes in perception of time and changes in visual perception. Paranoia, delusions and hallucinations may occur.

There's an increased risk of suicide not only for diagnosed patients but also for at-risk individuals.

Social skills and interpersonal abilities might be compromised. Some of these changes do not respond to pharmacological treatment and may configure a change in the individual's personality.

The dominant cognitive feature is executive dysfunction with difficulties in decision making, multi-tasking and more time needed to complete tasks.

Patients can be unaware of their cognitive, perceptual, motor and psychiatric problems.

\section{Management of Psychiatric Manifestations}

Patients with psychosis and/or disruptive behaviour may benefit from treatment with an antipsychotic such as quetiapine, olanzapine, risperidone or haloperidol (these may also help with chorea).

Depression can be effectively managed with a selective serotonin reuptake inhibitor.

There's no disease modifying treatment for HD. Therapy is focused on symptom management and supportive care and should be integrated in a multidisciplinary approach. A stable and calm environment is recommendable.

\section{Clinical Vignette}

Identification

M, male, 51 years old, married, with two children.

Previous Medical History

Huntington's Disease with clinical diagnosis when he was 37 years old. Medicated with Amantadine 200mg/day.

\section{Clinical History}

The assistant neurologist asked for a psychiatric consultation because his wife reported that lately he was more quarrelsome, sometimes being verbally aggressive with her and their children. He was also very jealous of her and would not let her use specific outfits. He never behaved like this before.

Also, he could not cope with unpredictability, becoming very anxious when things didn't occur according to plans. We was not able to make decisions like before, becoming detached from familiar plans. These characteristics turned him into "someone different", as his wife described.

During psychiatric evaluation he showed depressive and anxious symptoms and reported sexual dysfunction.

He was medicated with Escitalopram up to $20 \mathrm{mg}$ and Quetiapine 50mg. A month later he showed improvement in his depressive and anxious symptoms, as well as in his ideas of jealousy. However, he continued to have executive dysfunction, with difficulty in decision making and inability to deal with unpredictability.

\section{Results and Conclusion}

Physicians who deal with patients with Huntington's Disease should be aware that psychiatric manifestations are frequently present and may even precede motor symptoms.

Cognitive and behavioural changes place the greatest burden on the affected families and are highly associated with functional decline.

Therefore, they should be addressed in order to promote the patient's well being and autonomy. However, some of these manifestations can persist throughout the history of the disease.

Comprehension of first symptoms, described by some authors as prodromic, such as subtle motor changes, cognitive or behavioural symptoms, may allow future trials of therapies regarding the delay of clinical onset. 